\title{
The XUV split-and-delay unit at beamline BL2 at FLASH
}

\author{
M Wöstmann ${ }^{1}$, R Mitzner ${ }^{2}$, T Noll ${ }^{2}$, S Roling ${ }^{1}$, B Siemer ${ }^{1}$, F Siewert ${ }^{2}$, \\ S Eppenhoff ${ }^{1}$, F Wahlert ${ }^{1}$ and $H$ Zacharias $^{1}$ \\ ${ }^{1}$ Physikalisches Institut, Westfälische Wilhelms-Universität, Wilhelm-Klemm-Straße 10, \\ D-48149 Münster, Germany \\ ${ }^{2}$ Helmholtz-Zentrum Berlin für Materialien und Energie, Albert-Einstein-Straße 15, D-12489 Berlin, \\ Germany \\ E-mail:michael.woestmann@wwu.de
}

\begin{abstract}
For time-resolved extreme ultraviolet (XUV) pump-XUV probe experiments at the Free electron LASer in Hamburg (FLASH), a split-and-delay unit (SDU) has been built. It is implemented in beamline BL2 which provides a focal spot size of about $20 \mu \mathrm{m}$ diameter in the experiment. The beam is divided geometrically into two paths which can be delayed from -3 to +15 ps with respect to each other. The transmission up to $200 \mathrm{eV}$ photon energy is above $35 \%$ in one beam path and $74 \%$ in the other. The latter transmits the XUV beam again from 305 to $570 \mathrm{eV}$ (>1\% transmission). Thus almost the whole spectral range at FLASH is covered by the SDU with reasonable transmission, including the option to transport high-energy third harmonic radiation in one of the beam paths. Both beam paths are realigned into the original direction of the radiation at the end of the SDU. Thus the utilization of the divided as well as the original beam is enabled by simply moving the optical elements of the SDU into or out of the beam. Using the SDU, the coherence length and the average pulse duration at FLASH was determined to be $0.9-1.8 \mu \mathrm{m}$, depending on the wavelength, and about $30 \mathrm{fs}$, respectively, for the specific electron bunch parameters.
\end{abstract}

\section{Introduction}

The Free electron LASer in Hamburg (FLASH) was the first free electron laser (FEL) generating radiation in the extreme ultraviolet (XUV) and soft x-ray range of the electromagnetic spectrum from about $26 \mathrm{eV}$ to currently $300 \mathrm{eV}$ in the fundamental $[1,2]$. In addition, odd harmonics are generated at lower intensities. Thus photon energies up to $900 \mathrm{eV}$ are produced by the third harmonic. In recent years, much effort has been devoted to the development and completion of new FELs operating at wavelengths down to the Angstrom regime, which enables structural investigations on the atomic level. The Linac Coherent Light Source [3] and the SPring-8 Angstrom Compact free electron LAser [4] already operate in the hard $\mathrm{x}$-ray range with photon energies up to $20 \mathrm{keV}$. The high intensity of FEL radiation, many orders of magnitude higher than that from conventional sources in this spectral range, in combination with short pulses opens various new opportunities in research. Among these are nonlinear processes in the interaction of short wavelength radiation with matter at very high intensities up to $10^{16} \mathrm{~W} \mathrm{~cm}^{-2}$. Unexpected results were observed in multiple ionization of rare gas atoms $[5,6]$ and clusters [7, 8], as well as in saturated absorption of the $\mathrm{L}$ shell transition in $\mathrm{Al}$ [9]. Furthermore, diffractive imaging attracts a lot of interest [10] because the high pulse intensities allow us to investigate even uncrystallized probes such as biological molecules which are difficult to crystallize.

Since single-pass FELs such as FLASH typically provide short pulses of the order of 10 to $100 \mathrm{fs}$, dynamic processes occurring on a sub-ps temporal scale can be investigated. This includes the formation and dissociation of chemical bonds or the relaxation of clusters. Ultrafast dynamic processes are usually investigated by pump-and-probe experiments requiring two temporally separated pulses. A first pulse stimulates the process, while a second one samples the system at a defined temporal delay. Obviously, an optical laser can be 


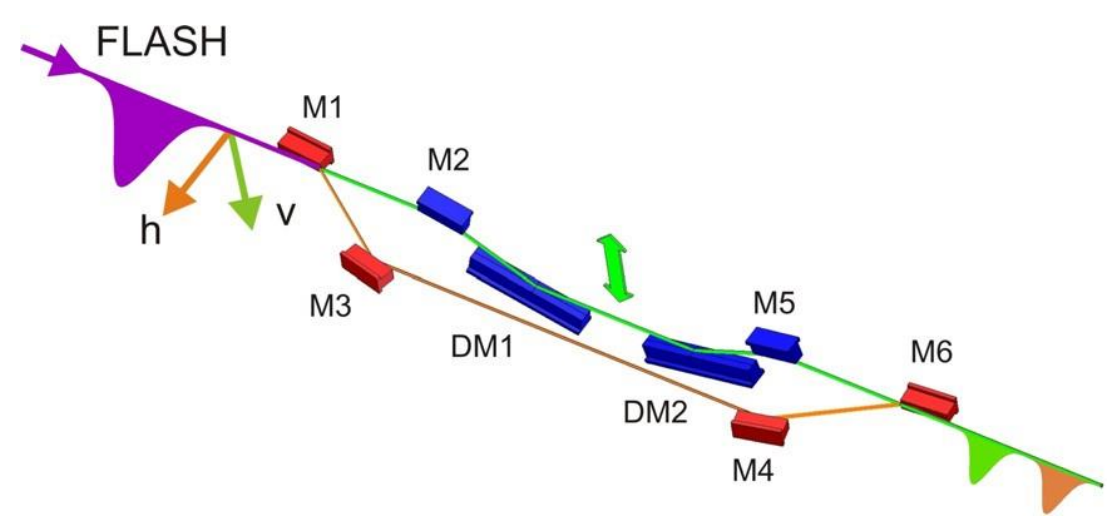

Figure 1. Sketch of the optical beam paths inside the SDU. The mirrors depicted in blue belong to the variable and the red mirrors belong to the fixed beam path. The beam is divided at the first mirror (M1) and recombined at the last mirror (M6). The longer mirrors in the middle (DM1 and DM2) are adjustable in height to set the delay. Adapted from [18].

synchronized with the XUV pulses generated by the FEL. At FLASH such an optical pumping is realized with a Ti:sapphire oscillator/amplifier system [11]. Currently, the accuracy of the synchronization between the XUV pulses from FLASH and the optical laser is limited to about 200 fs full-width at half-maximum (FWHM) ( $87 \mathrm{fs} \mathrm{rms}$ ) mainly due to the arrival time jitter of the electron bunches producing the XUV pulses [12]. By measuring the arrival time of the electron bunches from shot to shot, the actual time delay can be specified with sub-10 fs accuracy [13]. However, this requires subsequent analysis and sorting of every XUV pulse combined with the optical laser. Besides two-colour XUV-optical pump-andprobe experiments, for many applications it is attractive to have the high photon energy provided by the FEL for both the pump pulse and the probe pulse. In this case, a better temporal resolution with significantly reduced jitter between the pulses can be achieved by an XUV pulse split-and-delay unit (SDU). Additionally, two-colour XUV pump-XUV probe experiments using the fundamental and the third harmonic with photon energies even up to $570 \mathrm{eV}$ can be performed with such a device. At FLASH, two SDUs are realized: one in the plane grating monochromator beamline PG2 [14] and the other in the unmonochromatized, but focusable beamline BL2 which is described in this paper.

The performance of the SDU in BL2 has already been proved in various experiments such as holography $[15,16]$, cluster and plasma physics [17]. The SDU has further been used to characterize the properties of the XUV radiation via linear and nonlinear autocorrelation measurements. In this way, the coherence length could be determined to be $0.9-1.8$ $\mu \mathrm{m}$ at different wavelengths $[18,19]$ and the average pulse duration to be about 30 fs for the specific electron bunch parameters of FLASH at that time [20].

\section{Concept and design of the split-and-delay unit}

\subsection{General design}

The SDU was designed in 2004 in order to cover the spectral range up to the highest possible photon energy in the initial design of FLASH with reasonable transmission [21]. Only grazing incidence reflections at the mirrors provide a high transmission over a broad spectral range at these photon energies. The lack of broadband transmissive amplitude beam splitters, as readily available in the visible spectral range, requires the use of wavefront beam splitting. A delay in the range of a few tens of ps is desirable in order to follow the evolution of ultrafast dynamics of various systems. The schematic layout of the SDU is shown in figure 1. The XUV beam of FLASH is divided into two partial beams at the rear edge of the first mirror (M1). Each part is guided via different optical pathways and finally both beams are recombined again at the last mirror (M6). One of the optical paths, indicated by the blue colours of the mirrors in figure 1 , is variable in length, designed for a temporal delay from -3 ps up to +15 ps with respect to the other. Behind the SDU, the recombined beams follow the direction of the original beam which is necessary for integration into a beamline. Because of the grazing incidence reflections and the required delay range, the distance between the first mirror (M1) and the last mirror (M6) amounts to $1.3 \mathrm{~m}$.

\subsection{Beam paths and time delay}

Each optical path contains four mirrors guiding the beam through the SDU. An even number of reflections in every beam path ensures a correct reassembly of the beam profile behind the SDU. The variable channel is oriented in the vertical plane with two mirrors having a surface of $100 \mathrm{~mm} \times 25 \mathrm{~mm}$ and two larger ones with a surface of $250 \mathrm{~mm} \times 30 \mathrm{~mm}$, while the fixed channel is arranged horizontally using four $100 \mathrm{~mm} \times$ $25 \mathrm{~mm}$ mirrors. In the fixed beam path, the glancing angle of incidence at the mirrors amounts to $\alpha_{h}=3^{\circ}$. This angle is increased to $\alpha_{v}=6^{\circ}$ in the variable beam path in order to enable longer delays. The mirrors at the bottom of the variable path (DM1 and DM2) are adjustable in height and therefore enable the time delay. Upon movement, the point of beam impact onto the mirrors shifts along the mirror surface and thereby changes the optical path length. The time delay $f . . t$ achieved upon a height change $f . h$ can be calculated by

$$
f . . t=\frac{2}{c} f . . h \frac{\tan 2 \alpha_{v}}{\tan 2 \alpha_{v}-\tan \alpha_{v}}\left(\frac{1}{\sin 2 \alpha_{v}}-\frac{1}{\tan 2 \alpha_{v}},\right.
$$

where $c$ is the speed of light. Thus an alteration of the mirrors by $t . h=0.72 \mu \mathrm{m}$ results in a shift of $1 \mathrm{fs}$ in the optical pathway. 


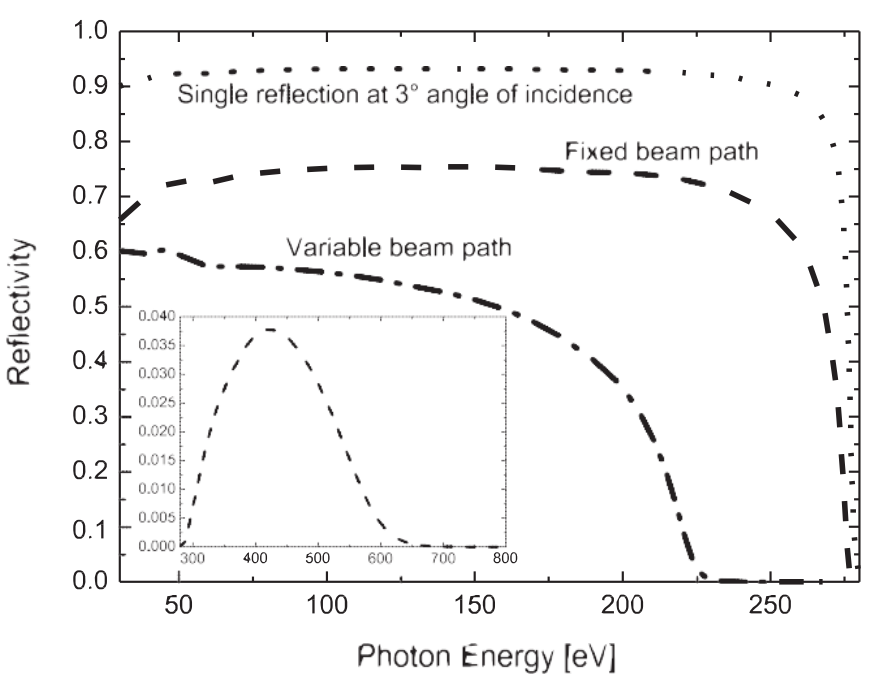

Figure 2. Calculated transmission of the SDU in each of the beam paths after four reflections. Inset: high photon energy transmission in the fixed beam path.

The incident XUV beam is split geometrically by placing the first mirror partly into the beam. A part of the incoming radiation is reflected sideways, while the remaining part passes the mirror unaffected. To minimize diffuse scattering, the irradiated edge should be very sharp. Different intensity ratios of the divided beams, which depend on the horizontal position of this mirror, can be realized by moving the whole SDU sideways.

For an alignment of the beam path, it is possible to tilt the first and the last mirror of each beam path (M1, M2, M5, M6) with the centre of rotation at the intensity maximum of the XUV pulse profile. For the fixed beam path, this point lies on the leading or rear edge of the mirrors. At the end of the SDU, the partial beams are recombined to the initial round beam except for a minor slit between them $(<1 \mathrm{~mm})$ to avoid contact of the variable beam with the leading edge of the recombination mirror (M6). In particular, the partial beams can easily be overlapped at different distances from the SDU by slightly tilting the last mirror (M6) in the horizontal plane.

\subsection{Mirrors}

The mirrors consist of a single crystal silicon substrate finished by deterministic surface treatment (ion beam finishing) [22] with a $40 \mathrm{~nm}$ thick diamond-like amorphous carbon coating [23]. The calculated reflectivity as a function of photon energy is shown in figure 2. For photon energies up to $200 \mathrm{eV}$ the transmission of the variable beam path is above $35 \%$, which covered the whole spectral range at FLASH when the SDU was designed. However, in recent years the electron beam energy of the linear accelerator has been increased to $1.25 \mathrm{GeV}$ and therefore the maximum photon energy is pushed up above the $\mathrm{K}$-edge of carbon at around $285 \mathrm{eV}$ where absorption processes deteriorate the reflectivity of the mirrors. For the fixed beam path, the $35 \%$ transmission mark is not reached until $270 \mathrm{eV}$. Changing the splitting ratio by passing more intensity through the variable beam path can offset unequal transmissions. The measured total transmission amounts to $48 \%$ at $52 \mathrm{eV}$ and $60 \%$ at $95 \mathrm{eV}$, which is lower than calculated primarily because diffraction losses originating from the beam splitting edge (M1) are not considered in the calculation. Interestingly, third harmonic radiation is transmitted again above the K-edge of carbon from about 305 to $570 \mathrm{eV}$, but only through the fixed beam path (see the inset of figure 2). Applying the fixed beam for the probe pulse enables two-colour XUV pumpXUV probe applications. In particular, the K-edges of $\mathrm{N}$ and $\mathrm{O}$ are accessible enabling time-resolved ESCA on important nitride and oxide compounds.

At a wavelength of $\lambda=13 \mathrm{~nm}$, the diameter of the XUV beam at FLASH at the entrance of the SDU is about $4.9 \mathrm{~mm}$ (FWHM) [24]. Assuming a Gaussian profile, this corresponds to a beam size of $10.4 \mathrm{~mm}$ within $5 \sigma$. As a consequence, the footprint of the radiation on the surface of a $100 \mathrm{~mm}$ long mirror irradiated at a glancing angle of $6^{\circ}$ is about 46.9 (FWHM) or $99.5 \mathrm{~mm}(5 \sigma)$. Since, depending on the splitting ratio, the original XUV beam is cut in half at mirror M1, all mirrors of the fixed beam path only have to catch a half profile on their long axis. At the corresponding glancing angle of $3^{\circ}$, half-profiles up to a width of $5.2 \mathrm{~mm}$ completely fit onto the mirror surfaces in this beam path. For longer wavelengths, the beam diameter as well as diffraction losses further increases.

A typical slope profile of one of the $100 \mathrm{~mm} \times 25 \mathrm{~mm}$ mirrors is shown in figure 3 . For this mirror, the rms slope error amounts to 0.04 arcsec. The other mirrors, including the larger $250 \mathrm{~mm} \times 30 \mathrm{~mm}$ mirrors, show similar profiles with slope errors less than 0.1 arcsec rms. The roughness amounts to :s:0.2 nm for every mirror as measured with a white light interferometer (magnification $20 \times$ and 50x). The excellent flatness of the mirrors is characterized by a radius of curvature of $100 \mathrm{~km}$ along the meridional mirror axis.

\section{Technical realization}

\subsection{General setup}

A cut through the construction of the SDU is shown in figure 4. Internal parts predominantly consist of sheet metal made from stainless steel in order to move vibrational resonances to high frequencies by realizing a good balance between stiffness and weight. The required stiffness is ensured by an octagonal optical bench made of $3 \mathrm{~mm}$ thick stainless steel (figure 4(7)). Onto this optical bench, the mirror holding modules are mounted. Because of the strong absorption of XUV radiation by air, the whole construction is embedded into a vacuum chamber (figure 4(8)). Two ion-getter pumps $\left(500 \mathrm{~s} \mathrm{~s}^{-1}\right.$ ) (figure 4(16)) are keeping the pressure inside the chamber at about $5 \times 10^{-9}$ mbar. All movements are enabled by flexure joints. If displacements are kept low, they have considerable advantages with respect to precision and repeatability, since they lack any mechanical slackness. The mirror holding modules further stiffen the optical bench as they serve as strutting.

\subsection{Mirror holders and delay stage}

The smaller mirrors (M1-M6) (figure $4(1,2)$ ) are attached to a basket consisting of 40 braces having flexure joints on 


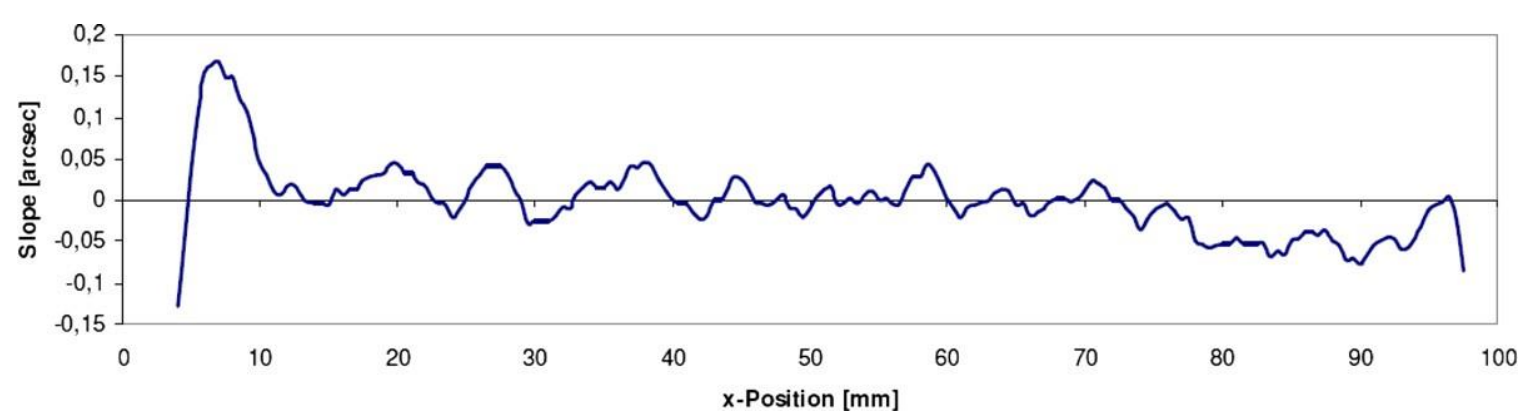

Figure 3. Slope profile of the long axis of a $100 \mathrm{~mm} \times 25 \mathrm{~mm}$ mirror taken on the centre lane. The profile results in a rms slope error of $0.04 \mathrm{arcsec}$ which corresponds to a residual figure error of $5.4 \mathrm{~nm}$ peak valley and $1.4 \mathrm{~nm} \mathrm{rms}$, respectively, and a radius of the curvature of $204 \mathrm{~km}$. The slopes were measured with the Nanometer Optical Component Measuring Machine (NOM) at BESSY [25].

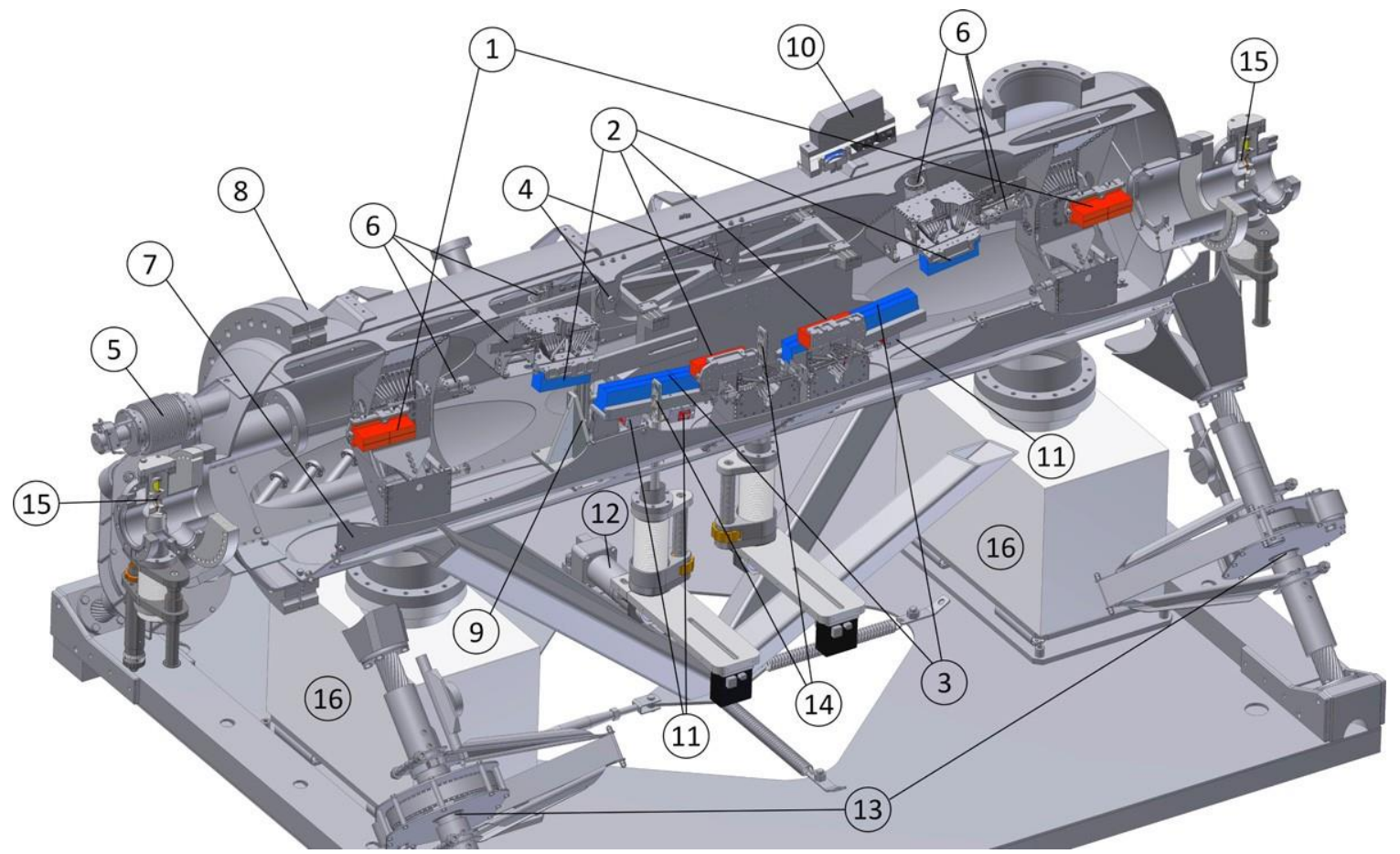

Figure 4. Illustration of the mechanical structure of the SDU. (1) Beam splitting and recombination mirrors M1 and M6, (2) beam guiding mirrors M2-M5, (3) delay setting mirrors DM1 and DM2, (4) guide rollers for the cable holding the delay stage, (5) stepper motor for the delay, (6) stepper motors for the guiding mirrors, (7) octagonal optical bench, (8) vacuum chamber, (9) linear encoder, (10) optical HeNe interferometer, (11) piezo actors for tilting compensation, (12) motor for torsion suppression, (13) telescopic legs with flexure ball joints, (14) retractable filter stages, (15) translation stages for beam observation, (16) ion getter pumps.

both sides. They are arranged in a way that the extensions of any brace meet at a single point located on the mirror surface and thus provide a gimbal movement of the mirror. When the joints are bent, tilting of the mirror surfaces occurs around this point with very little parasitic motion [26]. For all three rotatory degrees of freedom, a strong spring in combination with a movable cable holds the mirror in position. On the first and last mirror of each beam path (M1, M2, M5, M6), the relevant rotations are motor controlled, allowing adjustment from outside the vacuum chamber. Rotation around the surface normal is of minor importance in aligning the SDU and therefore the related movement is not equipped with motors. Using gear units in combination with stepper motors (figure 4(5)), the mirrors can be tilted with a resolution of the order of $1 \mu \mathrm{rad}$. The mirrors are mounted in a way that they turn around the maximum of the intensity distribution on their surface which is in the centre of the original beam. This implies that all mirrors of the fixed beam path, especially the beam splitting mirror (M1) and the recombination mirror (M6), are attached acentric to the basket.

Changing the delay is enabled by a concerted vertical movement of the long mirrors (DM1 and DM2) (figure 4(3)). This is realized by a parallelogram construction which again uses flexure joints on both ends of the upper and lower arms. The lateral faces of the parallelogram consist of two plates of aluminium. One plate is connected to the frame, and the other holds the mirrors. The mirrors are guided on a circular movement with a radius of $175 \mathrm{~mm}$, leading to a slight horizontal displacement which is easily covered by their width of $30 \mathrm{~mm}$. Normally, a tilting of the delay mirrors is avoided with this construction. The flexure joints consist of $0.1 \mathrm{~mm}$ sheet metal reinforced by a stranded wire. The sheet metal 
is necessary to ensure mechanical stability concerning lateral and compressive forces, while the stranded wire attenuates vibrations through friction between the laces. For the given geometry, a translation of about $13 \mathrm{~mm}$ is required to cover the entire delay range of 18 ps which is readily accessible by this construction. When the mirrors are in the most upper position, the variable beam path is shorter than the fixed path. This enables symmetric autocorrelation experiments because the temporal order of the XUV pulses can be inverted entirely. A cable guided in a loop by four rollers (figure 4(4)) is connected to the top and the bottom of the mirror holding plate. For setting the delay, this cable is attached to a stepper motor located outside the vacuum chamber (figure 4(5)). As shown in figure 7 , the maximal resolution of one full step of the motor corresponds to a vertical movement of the delay mirrors of about $0.03 \mu \mathrm{m}$, which results in a temporal shift of $40 \operatorname{arcsec}$ between the pulses.

\subsection{Delay determination}

Three independent systems to determine the actual delay position of the mirrors have been implemented. A linear encoder (figure 4(9)) (LIK 21, Numeric Jena) is installed in front of the parallelogram construction. Its accuracy amounts to $f . . h=0.05 \mu \mathrm{m}$. In addition, it possesses an internal reference mark making it an absolute measurement system. Secondly, the step position of the stepper motor can serve for the determination of the delay. A third system is an optical interferometer (SP 500, SIOS Meßtechnik) mounted outside the vacuum chamber (figure 4(10)). This system has the highest short-term accuracy of $0.1 \mathrm{~nm}$, but is only applicable for relative distance measurements. Additionally, it is affected by thermal expansion of the diameter of the vacuum chamber which is disadvantageous for determining the delay position over an extended period of time.

\subsection{External movement}

The whole vacuum chamber can be moved about five independent degrees of freedom for fine adjustment of the SDU to the beam and to move the mirrors out of the beam as well. Particularly, the splitting ratio is set by a simple horizontal displacement. Rotation around the long axis of the chamber is prevented, because the chamber is connected to the beam line by welded bellows at both ends which tolerate only very little torsion. A stepper motor (figure 4(12)) suppresses this rotation up to a few mrad. All other movements are realized by telescopic legs also driven by stepper motors (figure 4(13)). The flexibility is guaranteed by strong steel cords of $4 \mathrm{~cm}$ diameter for the connection to the chamber and the base plate, respectively. The accuracy and repeatability of the positioning are in the sub-mm range. By moving the chamber by about $15 \mathrm{~mm}$ vertically upwards and $20 \mathrm{~mm}$ horizontally to the right with respect to the FEL beam direction, all mirrors can be moved out of the FEL beam. At this position, there is a free space of $25 \mathrm{~mm}$ diameter where the XUV beam passes the SDU unaffected through the vacuum chamber allowing for other experiments.

\subsection{Beam diagnostics and filters}

For diagnostic purpose at four positions, screens can be moved into the beam paths. These positions are at the entrance and exit of the SDU (figure 4(15)), between the long mirrors of the delay stage and in front of the second mirror (M3) in the fixed beam path (figure 4(14)). For monitoring the beam position and the splitting ratio, phosphor (P43)-coated screens are employed, which are then imaged by CCD cameras (Basler A311f). Instead of the screens, it is also possible to position spectral filters into the beam. Filters applied in one of the beam paths enable two-colour pump-probe experiments with the fundamental and third harmonic of the FEL. In the separated beam paths there is room for three filters in addition to the screen in each of the translation shifts. At present, zirconium and aluminium are used to block the fundamental of $\lambda=$ $24 \mathrm{~nm}$ and the third harmonic at $\lambda=8 \mathrm{~nm}$, respectively. At the entrance and exit of the SDU, one filter is available in addition to the screen for general attenuation. Parylene is used in front and $\mathrm{Al}$ behind the SDU.

\section{Alignment and performance}

\subsection{Basic alignment}

Before applying it at FLASH, a pulsed optical laser was guided through the SDU for basic alignment. Furthermore, this laser was used for checking the correlation between the relative position of the delay mirrors (DM1 and DM2) and the resulting alteration in length of the optical beam path as calculated by equation (1). This was realized on a ps scale by simply recording the temporal position of the signal from an avalanche photodiode placed into the variable beam path behind the SDU. The photodiode was kept at saturation in order to keep the leading edge of the signal stable. By this method, the value of about 25 full steps of the stepper motor (figure 4(11)) for $1 \mathrm{fs}$ in the optical pathway was measured. This agrees well with the calculated mechanical movement of the delay mirrors by $f . . h=0.72 \mu \mathrm{m}$ in height for 1 fs delay, as shown in figure 7.

The point of zero delay at which both beam paths inside the SDU are of equal length is again found by an interference experiment. Since XUV FELs have a short coherence time of a few fs $[18,19]$, the visibility of the XUV interference fringes shows a pronounced maximum at zero delay. Figure 5 shows an example of interference patterns at $\lambda=8 \mathrm{~nm}$ and delays of $0 \mathrm{fs}$ and $10 \mathrm{fs}$, respectively. At zero delay, well-developed interference fringes which are on purpose slightly tilted can be noted, while they nearly disappear at a delay of $10 \mathrm{fs}$. Vertical fringes visible in the picture originate from diffraction at the beam splitting edge of the first mirror (M1) and the weaker circular intensity modulation is caused by diffraction from an aperture in front of the SDU. The evaluation of the pictures in figure 5 leads to the visibility distribution shown in figure 12 .

Figure 6 shows a snapshot of the recombined beams taken from the phosphor screen behind the SDU (figure 4(15)) at FLASH. The radiation directed through the fixed beam path appears on the left, while radiation passing through the variable beam path appears on the right. Except for minor 

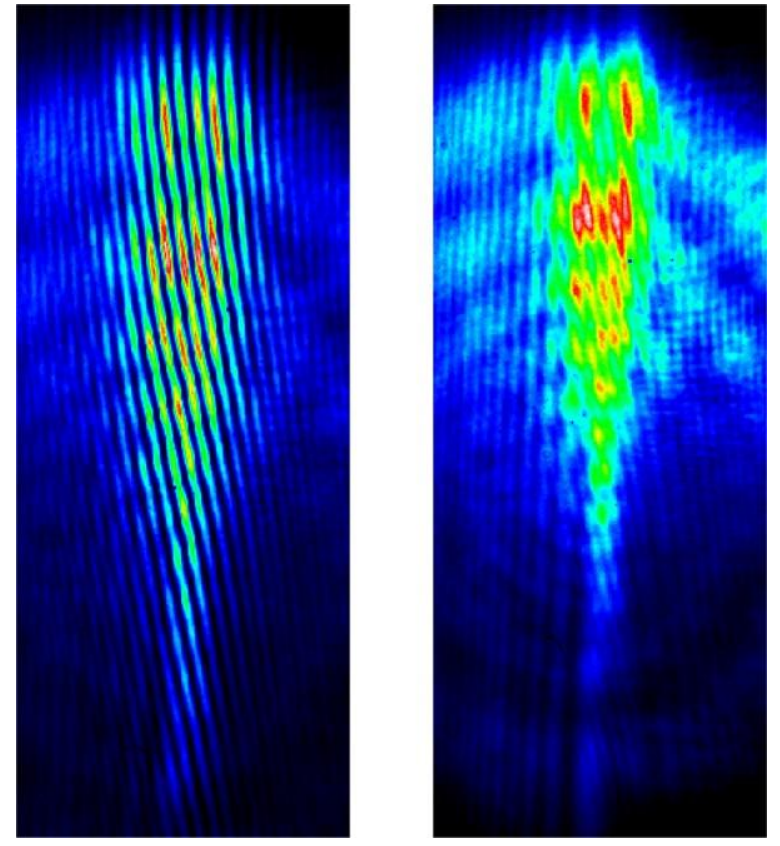

Figure 5. Interference patterns at a $\lambda=8 \mathrm{~nm}$ fundamental
wavelength of FLASH at a delay of $t . . t=0 \mathrm{fs}$ (left) and $t . . t=10 \mathrm{fs}$ (right). The visibility of the fringes deduced from the images shown fringes still visible in the right interferogram are associated with diffraction at the edge of the splitting mirror (M1).

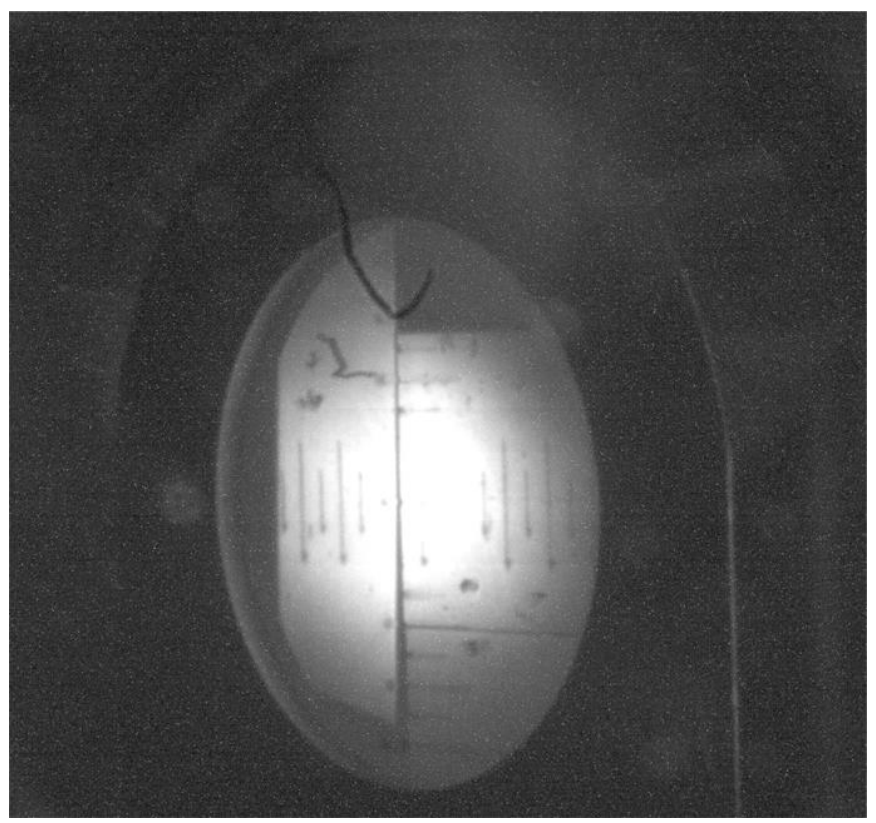

Figure 6. Image of the recombined partial beams taken from a retractable phosphor screen located behind the SDU. Long marks are separated by $1 \mathrm{~mm}$.

parts which are cut off at the mirrors, the partial beams form the initial round beam again. The lines visible in figure 6 are reference marks cut into the screen with a separation of $0.5 \mathrm{~mm}$ between two lines. Although some intensity is lost because of the limited length of the mirrors at photon energies above $95 \mathrm{eV}$, more than $5 \sigma$ of the original XUV beam fit onto the surface on their long axis. When the complete XUV beam

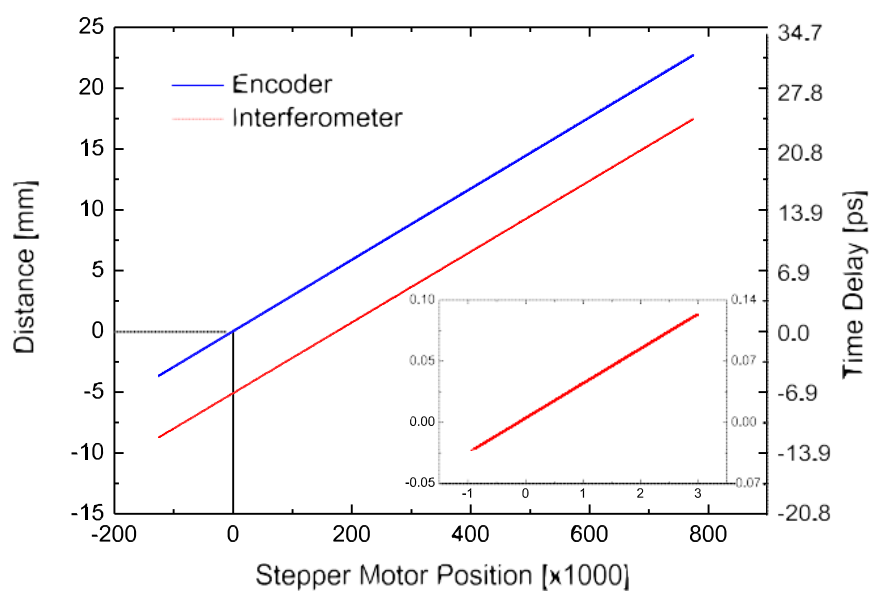

Figure 7. Distance values recorded by the interferometer and by the
encoder as compared to the position of the stepper motor for $f . . t=$ $36 \mathrm{ps}$. Three runs (back and forth) are shown. The zero-crossing of the encoder values equals a time delay of zero, while the interferometer values are shifted by $5 \mathrm{~mm}$ for better clarity. Inset: short range delay scan $(f . . t=160 \mathrm{fs})$ of the interferometer containing four runs (back and forth). The short range scan of the encoder is not shown due to a slight misalignment of the device.

of FLASH is guided through the SDU, a slight amount of radiation might pass the SDU directly without being reflected by the mirrors. To prevent this from happening, apertures of different diameters can be placed into the beam path before the SDU. The application of two apertures also serves for keeping the FEL pointing stable and thus a more constant splitting ratio from shot to shot is achieved as compared to without apertures.

\subsection{Accuracy and stability of the delay stage}

In figure 7, the travel distances recorded by the encoder and the interferometer are plotted against the position of the stepper motor. In total, $9 \times 10^{5}$ stepper motor positions have been followed resulting in a movement of $f . . h=26 \mathrm{~mm}$, which corresponds to a theoretical total time delay of $f . t=36 \mathrm{ps}$. Three runs (back and forth) including the whole delay range of the SDU are shown. Although the mechanics of the delay stage would enable much longer delays, the actual delay range is limited to about $18 \mathrm{ps}$ by the length of the delay mirrors (DM1 and DM2). At first, it is important to note that both detection systems record the same gradient of $0.029 \mu \mathrm{m} / \mathrm{step}$, which corresponds to about 40 arcsec/step. It has to be kept in mind that the interferometer records the relative distance along the surface normal of the second delay mirror (DM2) (figure 4(3)) which differs from the vertical shift in height by a factor of $\cos 6^{\circ}$. A mechanical backlash between the two directions of movement of the delay mirrors is observed which amounts to about $11 \mu \mathrm{m}$ corresponding to $15 \mathrm{fs}$ in time delay. With a deviation of $6.1 \mu \mathrm{m} \mathrm{rms} \mathrm{(8.5} \mathrm{fs)} \mathrm{and} 5.5 \mu \mathrm{m} \mathrm{rms} \mathrm{(7.6} \mathrm{fs),}$ respectively, from straight lines fitted to the data for each direction, the slopes show an excellent linearity over the whole range. The rms repeatability error by moving to a specific step position of the delay motor amounts to $1 \mathrm{fs}(0.72 \mu \mathrm{m})$. When the scanning range is reduced the repeatability error is further improved. The inset in figure 7 shows four runs (back and forth) recorded by the interferometer corresponding to $160 \mathrm{fs}$ 


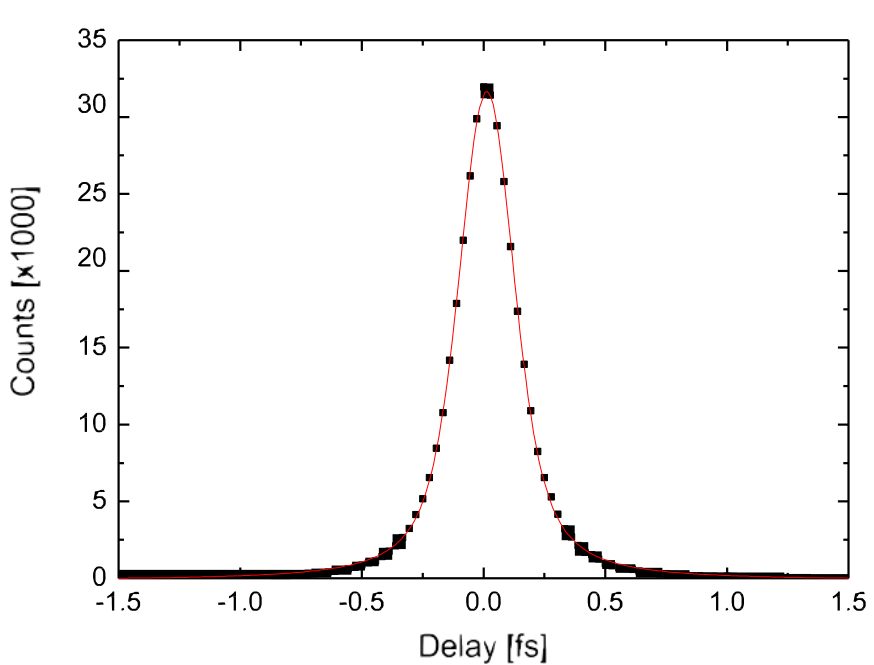

Figure 8. Histogram of the count rate taken from the interferometer at a nominal constant mechanical delay position. A Voigt profile is fitted to the data. The data were recorded for an interval of $8 \mathrm{~min}$ $30 \mathrm{~s}$, with a rate of $780 \mathrm{~Hz}$. The width amounts to $f . . t=280 \mathrm{as}$ FWHM.

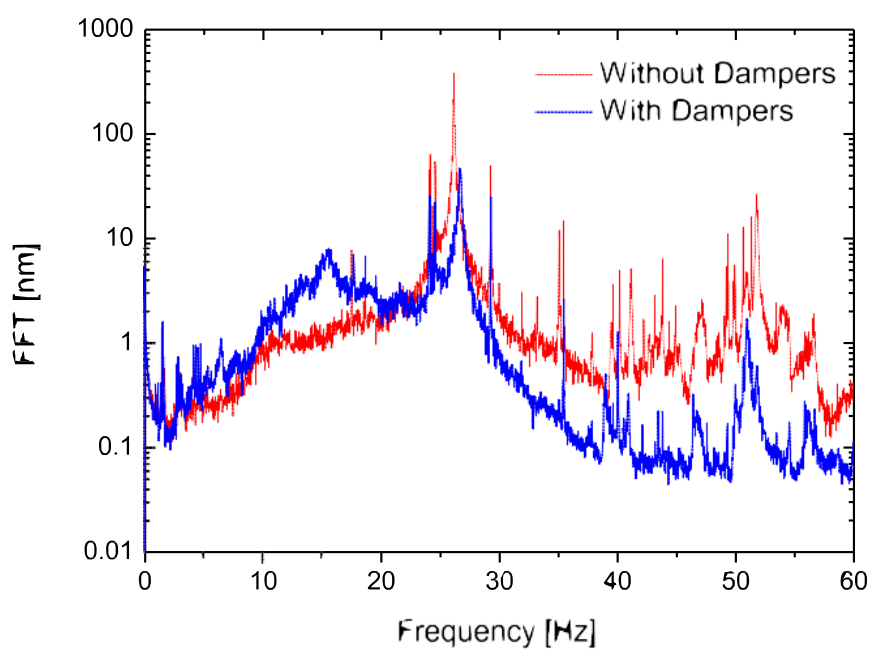

Figure 9. Averaged fast Fourier transformed (FFT) distance values of the delay mirrors taken from the interferometer. The total recording time was $8 \mathrm{~min} 30 \mathrm{~s}$.

in total time delay. In this case, the rms repeatability error amounts to $0.07 \mathrm{fs}(0.05 \mu \mathrm{m})$. In addition, the backlash is smaller amounting to only about $1.8 \mathrm{fs}(1.3 \mu \mathrm{m})$. The reason for this difference is currently under investigation.

The stability of the delay stage is illustrated in figure 8 . At a fixed nominal delay position the distance of the delay mirrors was recorded by the interferometer (figure 4(10)) with a sample rate of $780 \mathrm{~Hz}$ for a period of $510 \mathrm{~s}$. The resulting relative positions are binned into segments of $20 \mathrm{~nm}$ ( $\sim 30$ as) and then transformed into count rates which are plotted against the temporal delay. The FWHM of a fitted Voigt profile yields a delay variation of $f . . t=280$ as. This delay jitter arises from ground vibrations of the FLASH hall stimulating a resonance of the delay stage at $26.7 \mathrm{~Hz}$ which is apparent from figure 9, where the data record is Fourier transformed and the amplitudes are plotted versus the frequency. The red trace shows these variations in the case when the SDU is

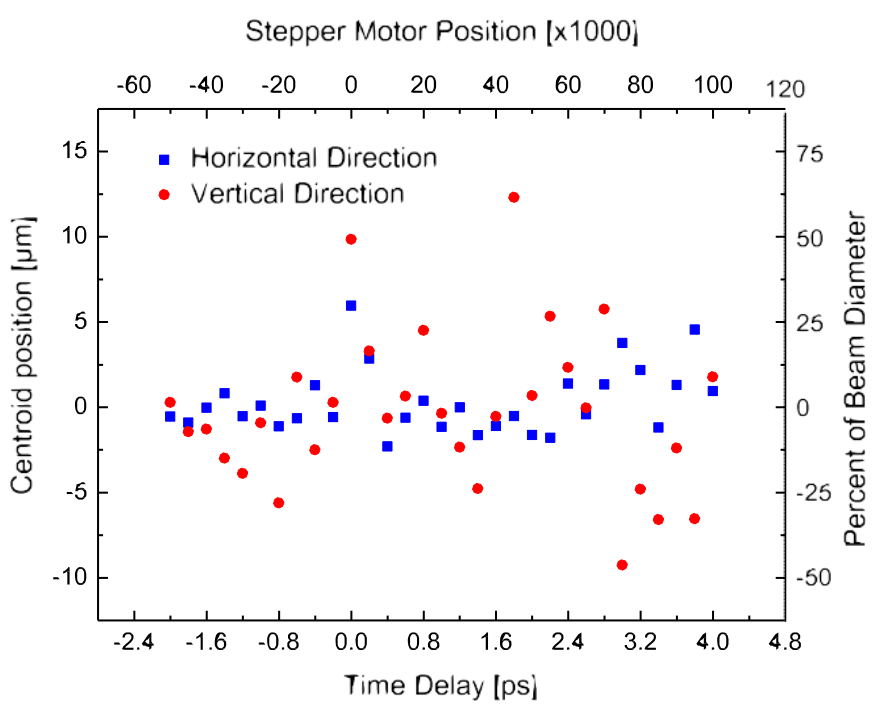

Figure 10. Single shot deviations of the centroid of the variable beam spot with respect to the centroid of the fixed beam spot during one delay scan. The horizontal difference is illustrated by blue squares, while the vertical difference is indicated by red dots. The beam diameter was assumed to be $20 \mu \mathrm{m}$. Over a delay range of $f . . t=6$ ps the rms spot variation amounts to $f . . x=1.9 \mu \mathrm{m}$ and $f . . y=$ $4.6 \mu \mathrm{m}$ for the horizontal and the vertical relative beam positions, respectively.

directly connected to the floor, and the blue curve when $33 \mathrm{~mm}$ thick passive damping units are inserted between the SDU and the supporting granite block. A strong reduction of the main amplitude by about a factor of 10 is observed. However, it should be noted that the exciting ground vibrations change permanently. The data sets used in figure 8 and that for the blue curve in figure 9 are identical.

\subsection{Pointing stability}

From the design of the delay stage, only a slight parasitic lateral movement of the delay mirrors (DM1 and DM2) should occur during displacement [26]. Unfortunately, they tilt causing a loss of spatial overlap in focused partial beams behind the SDU. This would impede any temporally resolved experiment, particularly if delays of several ps are requested. To compensate this movement, piezo actuators (figure 4(11)) (PU-500, Piezosystem Jena) operated in a closed-circuit mode are mounted below the mirrors. The actors are set with information from a feed-forward spreadsheet which must be prepared by manually overlapping the partial beams before the measurement. It should be mentioned that the closed-circuit mode of the piezo actors does not cause any wobble of the variable partial beam behind the SDU.

Applying a feed-forward spreadsheet containing general piezo values for every delay position was tried. However, experience has shown that this method does not yield a sufficient stability of the variable focal spot, mainly because the tilting of the delay mirrors (DM1 and DM2) is dependent on the delay range applied. Figure 10 illustrates the stability of the variable focal spot imaged on a Ce:YAG screen which was recorded by a CCD camera. The blue squares show the relative horizontal position of the centroid of the intensity 


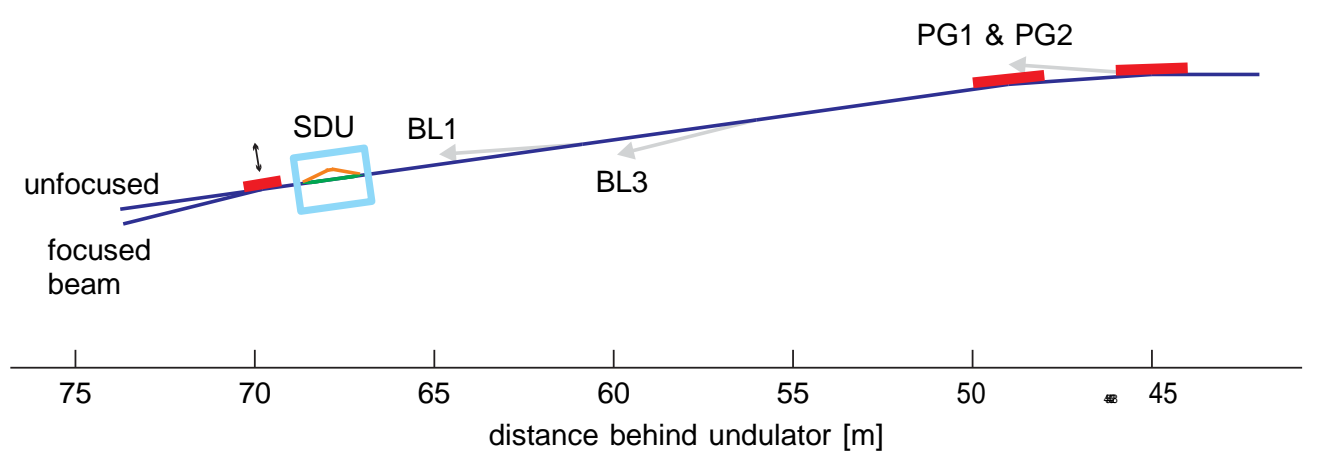

Figure 11. Beamline BL2 in the FLASH hall from above, The XUY beam from FLASH arrives from the right where it is deflected by two
mirrors at a 2 angle of incidence. The SDU is highlighted in light blue. Behind the SDU, a refocusing mirror $(f=2 \mathrm{~m})$ at a 3 angle of incidence can be moved into the beam. In addition, the branches to the plane grating beamlines (PG1 and PG2) and BL1 as well as BL3 are indicated.

distribution, while the red dots show these relative positions in the vertical direction. This measurement was performed before the dampers for vibration suppression were added which retrospectively had a big influence on the pointing stability of the variable focal spot, specifically on the vertical axis. Nevertheless, the spot remained over most part of the delay of $f . . t=6 \mathrm{ps}$ within $\pm 10 \%$ of the spot diameter. The horizontal and vertical variations of the focal spot amounted to $f . . x=$ $1.9 \mu \mathrm{m}(\mathrm{rms})$ and $f_{. .} y=4.6 \mu \mathrm{m}(\mathrm{rms})$, respectively.

The spreadsheet used in figure 10 was generated by observing the intensity centroids of the focused beams on the CCD camera. Most commonly a satisfying spatial overlap is achieved by simply controlling it visually at a few delay positions. It has to be kept in mind that randomly set delay positions and realignment have negative influence on the overlap of the focused partial beams. Hence, a new spreadsheet is generated with the knowledge of the time delay sequence before every experiment. In this way, a stable overlap over at least a whole day is ensured.

\section{First results}

\subsection{Beamline BL2 at FLASH}

The SDU is implemented into the beam transport system of FLASH at beamline BL2 as illustrated in figure 11. The position is about $68 \mathrm{~m}$ behind the last undulator segment. Behind the SDU, an elliptical refocusing mirror $(f=2 \mathrm{~m})$ can be moved into the beam generating a focal spot of $20 \mu \mathrm{m}$ (FWHM) diameter. Most experiments investigate dynamics using the focusing mirror in combination with the SDU since high photon densities are necessary. For example, experiments in cluster dynamics, warm dense matter dynamics, etc were performed with the SDU. In addition, also experiments using the unfocused beam, like holographic imaging, can be performed. At BL2 it is possible to utilize the synchronized optical laser as well. This could serve for pump-probe-probe experiments where probing is done at two delays or with two colours. Many diagnostic tools, apertures and a beam attenuator help the users managing their experiments [27].

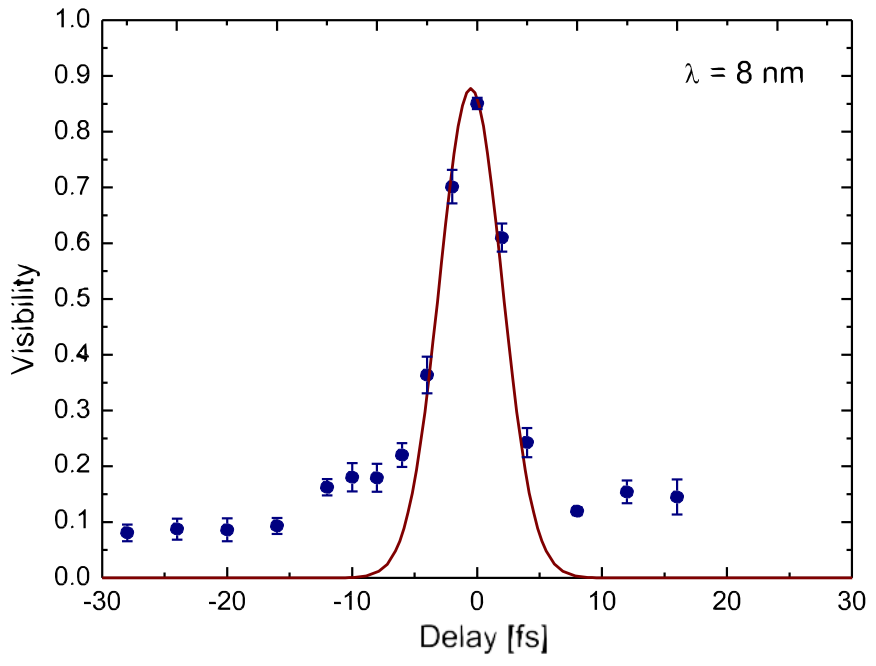

Figure 12. Visibility distribution of interference fringes at different time delays. The data were taken at FLASH at the fundamental at $\lambda=8 \mathrm{~nm}$. The blue dots show the calculated visibility averaged over ten individual pulses. The red curve is a Gaussian fit with a full-

\subsection{Coherence time and pulse duration}

At first, the SDU has been applied for the experimental determination of some parameters characterizing the XUV beam at FLASH. Among these are the coherence time and the average pulse duration. The coherence time was deduced from the visibility of the two beam interference fringes arising from simply overlapped partial beams directly on an XUV sensitive CCD camera. A visibility distribution at a wavelength of $8 \mathrm{~nm}$ is shown in figure 12, leading to a coherence time of $t_{c}=$ 2.9 fs. As mentioned before, such a measurement also serves for a precise determination of the zero delay position of the SDU. Because of the short wavelength a very small angle in the $\mu \mathrm{rad}$ range between the beams is necessary to resolve the interference fringes on the camera. This requires a long distance of up to $20 \mathrm{~m}$ between the SDU and the CCD camera. The determined coherence time depends on the wavelength, decreasing from $t_{c}=6 \mathrm{fs}$ at $32 \mathrm{~nm}$ to $t_{c}=2.9 \mathrm{fs}$ at $8 \mathrm{~nm}$ [19]. 


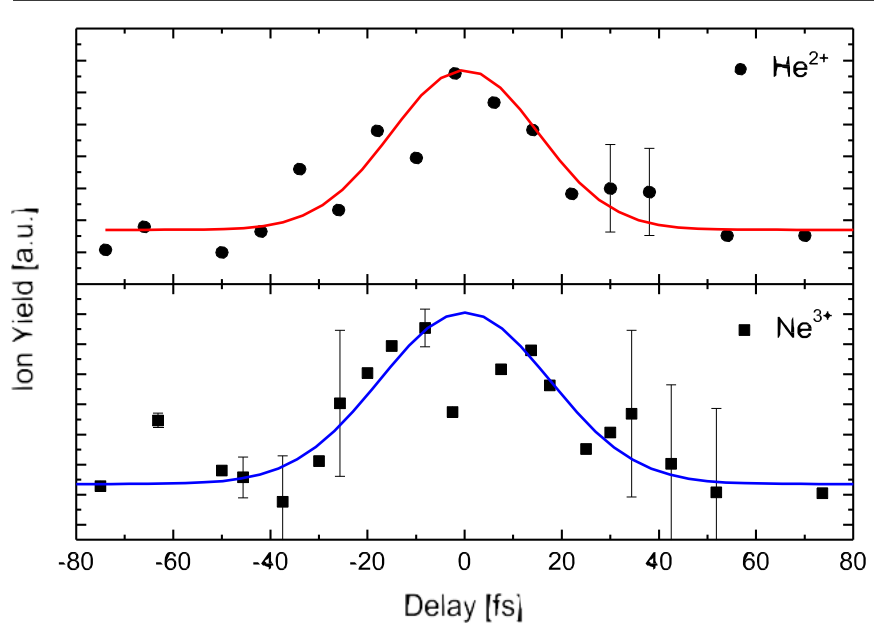

Figure 13. Yield of doubly charged $\mathrm{He}^{2+}$ ions and triply charged $\mathrm{Ne}^{3+}\left(\right.$ from $\mathrm{Ne}^{2+}$ ) after two-photon photoionization at different delays [20].

Since SASE FEL radiation consists of several independent longitudinal modes, the pulse duration is longer than the coherence time. For deriving the pulse duration, nonlinear processes have to be induced by the overlapping pulses. At a photon energy of $51.7 \mathrm{eV}$, the dominant channel in generating doubly charged $\mathrm{He}^{2+}$ ions is two-photon photoionization [28]. Similarly, triply charged $\mathrm{Ne}^{3+}$ ions are generated from $\mathrm{Ne}^{2+}$ by a two-photon process at the same photon energy. By detecting the $\mathrm{He}^{2+}$ and the $\mathrm{Ne}^{3+}$ ions, respectively, with a timeof-flight spectrometer at varying pulse delays, an intensity autocorrelation is recorded from which the pulse duration can be derived. Figure 13 shows the detected average ion yields for $\mathrm{He}^{2+}$ and $\mathrm{Ne}^{3+}$, which was recorded in another beamtime period, versus pulse delay. Assuming a Gaussian temporal pulse shape, an average pulse duration of about $30 \mathrm{fs}$ can be derived in either case (red lines). The introduction of a third harmonic acceleration module at FLASH in 2010 had a significant influence on the charge distribution of the electron bunches [29, 30], yielding a more reproducible electron bunch profile where a larger fraction of the overall charge contributes to the lasing process. This in turn resulted in a higher radiated power but adversely affected the pulse duration which is extended to about 70-200 fs (FWHM).

\section{Summary}

A split-and-delay unit (SDU) was built for FLASH which is now implemented at BL2. This device is designed for a temporal delay from -3 to 15 ps between the two pulses with an accuracy in the sub-fs regime. The fundamental of the XUV pulses is transmitted up to photon energies above $200 \mathrm{eV}$, while two-colour pump and probe experiments are possible with photon energies up to $570 \mathrm{eV}$. The performance of the SDU has been demonstrated in a number of applications such as autocorrelation measurements, cluster and warm dense matter experiments and holography.

\section{Acknowledgments}

The support of the FLASH team and the financial support by the Bundesministerium für Bildung und Forschung, project 05KS4PMC/8, within the priority research program FSP301 'FLASH' are gratefully acknowledged by the authors. The authors thank A A Sorokin for his support in taking ion timeof-flight data of ionized rare gases.

\section{References}

[1] Ackermann W et al 2007 Nature Photon. 1336-42

[2] Schreiber S et al 2011 Status of the FEL user facility FLASH FEL2011: Proc. Int. Free Electron Laser Conf. (Shanghai, China) TUPB04 (http://accelconf.web.cern.ch/AccelConf/ FEL2011/papers/tupb04.pdf)

[3] Emma P et al 2010 Nature Photon. 4 641-7

[4] Tanaka H et al 2012 Nature Photon. 6 540-4

[5] Sorokin A A et al 2007 Phys. Rev. Lett. 99213002

[6] Richter M et al 2009 Phys. Rev. Lett. 102163002

[7] Wabnitz H et al 2002 Nature 420 482-5

[8] Bostedt C et al 2008 Phys. Rev. Lett. 100133401

[9] Nagler B et al 2009 Nature Phys. 5 693-6

[10] Chapman H N et al 2006 Nature Phys. 2 839-43

[11] Redlin H et al 2011 Nucl. Instrum. Methods A 635 S88-93

[12] Grguraš I et al 2012 Nature Photon. 6 852-7

[13] Tavella F et al 2011 Nature Photon. 5 162-5

[14] Sorgenfrei F et al 2010 Rev. Sci. Instrum. 81043107

[15] Pfau B et al 2010 New J. Phys. 12095006

[16] Günther C M et al 2011 Nature Photon. 5 99-102

[17] Krikunova M et al 2012 J. Phys. B: At. Mol. Opt. Phys. 45105101

[18] Mitzner R et al 2008 Opt. Express 16 19909-19

[19] Roling S et al 2011 Phys. Rev. ST Accel. Beams 14080701

[20] Mitzner R et al 2009 Phys. Rev. A 80025402

[21] Mitzner R et al 2005 Proc. SPIE 5920 59200D

[22] Schindler A et al 2004 Proc. SPIE 5180 64-72

[23] Störmer M 2008 Proc. SPIE 7077707705

[24] The TESLA Test Facility FEL Team 2002 SASE FEL at the TESLA Facility, Phase 2 Technical Report TESLA-FEL 2002-01 (Deutsches Elektronen-Synchrotron, DESY) (http://flash.desy.de/sites2009/site_vuvfel/content/e403/ e1642/e750/e751/infoboxContent752/fel2002-01.pdf)

[25] Siewert F et al 2012 Opt. Express 20 4525-36

[26] Noll Tet al 2009 Precis. Eng. 33291304

[27] Tiedtke K et al 2009 New J. Phys. 11023029

[28] Nikolopoulos L A A and Lambropoulos P 2006 J. Phys. B: At. Mol. Opt. Phys. 39 883-93

[29] Vogel E et al 2010 Test and commissioning of the third harmonic RF system for FLASH IPAC10: Proc. Int. Particle Accelerator Conf. (Kyoto, Japan) THPD003 (http://accelconf.web.cern.ch/Accelconf/IPAC10/papers/ thpd003.pdf)

[30] Edwards $\mathrm{H}$ et al $20103.9 \mathrm{GHz}$ cavity module for linear bunch compression at FLASH LINAC2010: Proc. Linear Accelerator Conf. (Tsukuba, Japan) MO304 (http://accelconf.web.cern.ch/Accelconf/LINAC2010/ papers/mo304.pdf) 